\title{
Molecular x-ray computed tomography of myelin in a rat brain
}

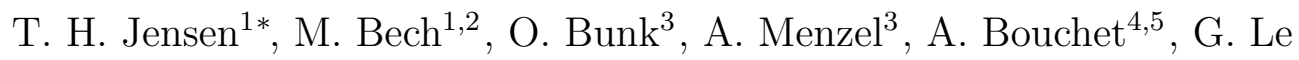 \\ Duc $^{4}$, R. Feidenhans' $1^{1}$, F. Pfeiffer ${ }^{2}$ \\ 1 Niels Bohr Institute, University of Copenhagen, Copenhagen, Denmark \\ 2 Department of Physics, Technische Universität München, Garching, Germany \\ 3 Paul Scherrer Institut, Villigen PSI, Switzerland \\ 4 European Synchrotron Radiation Facility, Grenoble, France \\ 5 Grenoble Institute of Neurosciences (Team 7), Grenoble, France
}

\begin{abstract}
In this work we demonstrate the feasibility of applying small-angle x-ray scattering computed tomography (SAXS-CT) for non-invasive molecular imaging of myelin sheaths in a rat brain. Our results show that the approach yields information on several quantities, including the relative myelin concentration, its periodicity, the total thickness of the myelin sheaths, and the relative concentration of cytoskeletal neurofilaments. For example the periodicity of the myelin sheaths varied in the range from 17.0 to $18.2 \mathrm{~nm}$ around an average of $17.6( \pm 0.3) \mathrm{nm}$. We believe that imaging, i.e., spatially resolved measuring these quantities could provide general means for understanding the relation to a number of neurodegenerative diseases.
\end{abstract}

Keywords: Molecular tomography, X-ray scattering, Myelin, X-ray computed tomography

*Corresponding author.

Email addresses: torbenj@fys.ku.dk (T. H. Jensen $\left.{ }^{1}\right)$, oliver.bunk@psi.ch (O. Bunk $^{3}$ ), franz.pfeiffer@ph.tum.de (F. Pfeiffer $\left.{ }^{2}\right)$

This document is the accepted manuscript version of the following article:

Jensen, T. H., Bech, M., Bunk, O., Menzel, A., Bouchet, A., Le Duc, G., ... Pfeiffer, F. (2011). Molecular X-ray computed tomography of myelin in a rat brain. NeuroImage, 57(1), 124-129. https://doi.org/10.1016/j.neuroimage.2011.04.013

This manuscript version is made available under the CC-BY-NC-ND 4.0

1icense http://creativecommons.org/1icenses/by-nc-nd/4.0/ 


\section{Introduction}

Myelin sheaths are lamellar membranes that wrap around neuronal axons. The sheaths are important for the central nervous system as they ensure rapid and non-interrupted communication of signals along axons. Structural changes of the myelin sheaths have been associated with a number of widespread neurodegenerative diseases such as cerebral malaria (Janota and Doshi, 1979), multiple sclerosis (Prineas, 1985; Peterson et al., 2001; Trapp et al., 1998) and Alzheimer's disease (Bartzokis et al., 2004). The origin and evolution mechanisms of the diseases are still largely not understood, but it is hypothesized that it is correlated with changes in the myelin formation and structure. A better understanding of the changes in the myelin sheath structure and its correlation to the pathologies is important for the development of methods for prevention or treatment of the diseases.

The changes in the myelin sheaths have been studied with a number of methods such as light (Trapp et al., 1998) and electron microscopy (Karlsson, 1966; Sjöstrand, 1953), magnetic resonance imaging (Hui et al., 2010) and x-ray diffraction (De Felici et al., 2008). All of these methods provide insight into the concentration and molecular structure of the myelin. While myelin sheaths from different types of nerves and in several different animal models have been extensively studied (Karlsson, 1966; Marta et al., 2003), no mapping of the variations of the molecular structure of myelin sheaths in a complete brain has been achieved. In this paper we introduce a method to visualize molecular variations of the myelin structure in a rat brain. Our approach is based on the fusion of two well-established biomedical investigation methods: Small-angle x-ray scattering (SAXS) and computed tomography $(\mathrm{CT})$. Combined with a specially developed data processing schemes, our approach delivers non-invasive volume-resolved studies of the molecular variations of the myelin sheaths in a whole rat brain.

SAXS-CT has been demonstrated previously in a few cases with soft matter and biological samples (Harding et al., 1985, 1987, 1989; Kleuker et al., 1998; Schroer et al., 2006; Stock et al., 2008). However the combination of SAXS and CT requires the acquisition of vast amounts of data. Previous studies have as a consequence been limited to a comparatively low resolution. The development of fast and essentially noise-free detectors, bright sources and automated analysis schemes have facilitated the efficient acquisition and processing of the hundreds of thousands of SAXS patterns needed for the high resolution SAXS-CT studies necessary for molecular imaging. 
(a)

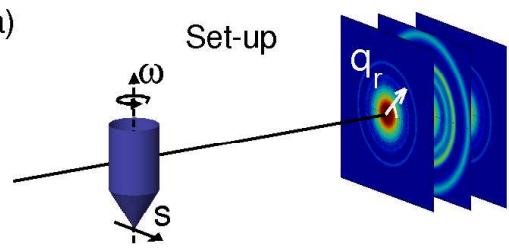

(c)

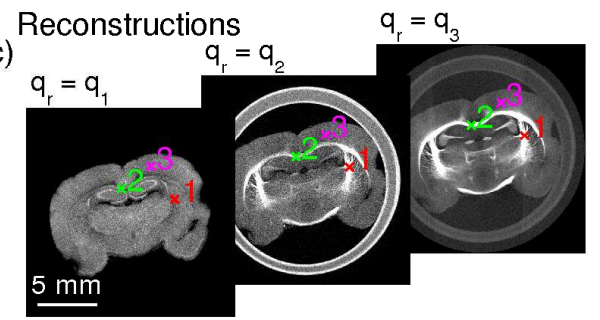

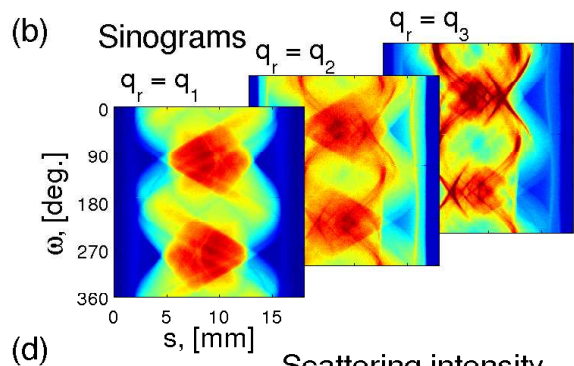

(d)

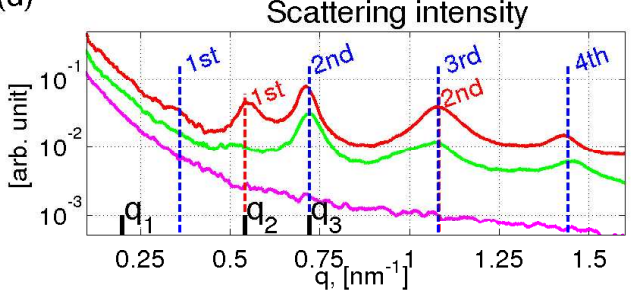

Figure 1: Experimental set-up and principle of SAXS-CT. (a) Using a pencilbeam( $1^{\text {st }}$ generation CT geometry), scattering patterns are collected by translating the sample through the beam, $s$, at different orientations, $\omega$. (b) The scattering patterns are azimuthally averaged and for each value of $q_{r}$ sinograms are generated. (c) These sinograms are used to reconstruct the differential scattering cross section for the associated value of $q_{r}$. (d) When the reconstructions are made it is then possible to extract complete scattering curves for all voxels in the sample, by combining the information from the slices reconstructed for each value of $q_{r}$. The curves shown here correspond to the points marked in (c). Bragg peak series from myelin sheaths (blue) and cytoskeletal neurofilaments (red) are marked in $(\mathrm{d})$.

Scanning based 2D SAXS mapping of bone and tissues have also been performed (Fratzl et al., 1997; Rinnerthaler et al., 1999; Paris et al., 2000; Fernandez et al., 2005; Gourrier et al., 2007; Bunk et al., 2009; Müller et al., 2010). These experiments provide 2D structural information, but often require destructive sample preparation and provide no depth-resolved information about the sample.

\section{Materials and methods}

\subsection{SAXS-CT methodology and data processing}

Figure 1 shows the experimental set-up and principle of SAXS-CT. 2D SAXS patterns are recorded using a pencil beam. This is repeated for each horizontal sample translation, $s$, and rotation, $\omega$, such that 2D SAXS patterns are collected point by point (Fig. 1a). In this way a full tomographic 
data set is collected point by point using a 1st generation tomography approach. The 2D SAXS patterns are azimuthally averaged and sinograms are generated for each value of $q_{r}$ (Fig. 1b). $q_{r}$ refers to the radial part of the momentum transfer. Each sinogram is used to reconstruct the differential scattering cross section for the associated value of $q_{r}$ (Fig. 1c). Once the reconstructions have been made for all values of $q_{r}$ it is possible to tomographically extract full scattering curves in a non-destructive manner for all voxels in the sample (Fig 1d).

Using the scattering curves we obtain information about the brain at the nano-scale. The momentum transfers of the scattering curves correspond to real space distances of 3-60 $\mathrm{nm}$. With SAXS-CT we are thus probing length scales that are smaller than the voxel size.

\subsection{Data collection}

The SAXS-CT measurements were performed at the cSAXS beamline (Bunk et al., 2009) of the Swiss Light Source. The following parameters were used for the data acquisition: $\mathrm{X}$-ray energy $18.58 \mathrm{keV}$, with $\Delta E / E \approx 2 \cdot 10^{-4}$, intensity of $10^{11}$ photons/second, focus spot size $25 \times 25 \mu \mathrm{m}^{2}$, sample to detector distance $7117 \mathrm{~mm}$. The scattering was recorded using a single photon counting PILATUS 2M detector (Henrich et al., 2009). The PILATUS 2M detector has $1475 \times 1679$ pixels of $172 \times 172 \mu \mathrm{m}^{2}$ resulting in an active area of $253.7 \times 288.8 \mathrm{~mm}$. The PILATUS $2 \mathrm{M}$ detector is essentially noise free and yields excellent statistics, with useful azimuthally averaged photon counts as low as 0.1 photons/pixel. The sample was imaged from 541 equidistant angles over 360 degrees. 720 scattering patterns were collected for each angle using a step size of $25 \mu \mathrm{m}$. The exposure time was $150 \mathrm{~ms}$ for scattering measurements and $10 \mathrm{~ms}$ for absorption measurements. The total acquisition time for the scattering and absorption measurements for one tomographic slice approximately 24 hours. A total of 390'061 scattering patterns were collected per slice. No beam damage was observed in any of our samples.

\subsection{Data processing}

The 2D scattering patterns were azimuthally averaged using a customized in-house developed code written in Matlab. After correcting for absorption reconstructions were made for 1152 different values of momentum transfer using a standard filtered backprojection algorithm (Kak and Slaney, 1988). The momentum transfers were calculated from the geometry of the setup and the energy of the x-rays (Als-Nielsen and McMorrow, 2001). Since all 
scattering occurs at small angles, the samples are limited in size and the absorption varies smoothly, the scattered photons can be assumed to exit the sample along the same path as the transmitted photons. It is thus possible to separate the absorption and scattering terms, and thus appropriate to use standard backprojection algorithms for the reconstructions.

In our reconstruction algorithm we assume that the local differential scattering cross section is independent of the sample orientation. It is known that the myelin sheaths are aligned along the fiber directions in the brain. We however sample relatively large volumes $\left(25 \times 25 \times 25 \mu \mathrm{m}^{3}\right)$, and assume that the scattering is locally isotropic. We have not seen any artifacts related to preferential orientation of the myelin sheaths. Preferential orientation of the myelin sheaths may influence the determined concentration of myelin.

\subsection{Sample preparation}

The rat brain used for this study, was a tumor inoculated brain (for another study). However the rat was euthanized on day 7 , so we would expect only a very small tumor. No tumor was imaged, and the tumor type does not to our knowledge interfere with the myelin sheaths. The preparation of the brain took place as follows. Immediately following euthanization the brain was sampled and fixed in $10 \%$ formalin. The fixation duration was minimum 10 days prior to any experiment. All operative procedures related to animal care strictly conformed to the Guidelines of the French Government with licenses 380825 and B3818510002 and they were reviewed by the Internal Evaluation Committee for Animal Welfare and Rights of the ESRF.

\section{Results}

\subsection{Mapping the myelin sheath concentration}

To demonstrate the potential of the method, particularly for biomedical applications, a formalin-fixed rat brain was investigated using SAXS-CT. Figure 1c shows an example of a reconstruction of such a brain with a few associated reconstructed scattering patterns displayed in Figure 1d. In the scattering patterns we note several distinct Bragg peaks. One of the series of Bragg peaks is associated with the quasi periodical arrangement of the lamellar structure of the myelin sheaths (De Felici et al., 2008; Tartari et al., 2009; Kirschner et al., 2010).

The peaks provide information about the molecular organization of the myelin. 
In point 1 (Fig. 1c,d) a second Bragg peak series is present. This second series is probably associated with the cytoskeleton neurofilaments (De Felici et al., 2008; Tartari et al., 2009). In the following we will further analyze the two Bragg series. Two different coronal slices from the brain are presented in Fig. 2. In the first row maps of the integrated scattering cross section for $q_{r}=[0.05-1.4] \mathrm{nm}^{-1}$ are shown. The scattering cross section is a term used in x-ray scattering which describes the samples ability to scatter x-rays in a specific direction. The panels clearly show the overall anatomy of the imaged brain slices and are displayed to provide anatomical references to which we can relate the myelin maps.

Knowing the full scattering curve in each voxel, we fitted the intensity, position and width of the second-order Bragg peak associated with the myelin series. The peak was fitted with a gaussian profile with a linear background. The intensity of the peak is proportional to the amount of myelin in each voxel, which represents a volume of $25 \times 25 \times 25 \mu \mathrm{m}^{3}$ (Als-Nielsen and McMorrow, 2001; Kirschner et al., 2010). The middle row of Fig. 2 displays the myelin concentration as calculated from the peak intensity. Note that the accuracy map of the myelin concentration is only accurate if the scattering is locally isotropic. In Fig. 2c the myelin is mainly associated with the corpus callosum(cc), external capsule(ec), the anterior commisure(ac), as well as the fornix(f). A somewhat lower concentration of myelin is associated with the striatum(st) and the optic chiasm(ox). In Fig. $2 d$ we again note the myelin associated with the corpus callosum and the external capsule. The myelin in the internal capsule(ic), as well as some of the connections between the internal and external capsule are also visible. Finally we note the myelin in the fimbria(fi) of the hippocampus as well as in the habenula(Hb) and the optical tract(opt). These maps of the myelin concentration correlate well with the location and concentration of myelin known from anatomy and histology (Mikula et al., 2007).

The method of SAXS-CT is highly selective. The myelin sheaths are the only tissue in the brain with a well-defined repetition unit of $\sim 17.6 \mathrm{~nm}$ and has therefore been used in previous, not spatially resolved studies byas well De Felici et al. (2008). Other methods such as e.g. histology can also selectively image myelin. With histology it is possibly to stain for and image specific molecules, such as myelin basic protein (MBP). It is however associated with great difficulty to quantify the concentration of these proteins. X-ray CT using osmium tetroxide staining has also been used for imaging the spatial location of myelin sheaths (Lareida et al., 2009). The method however pro- 

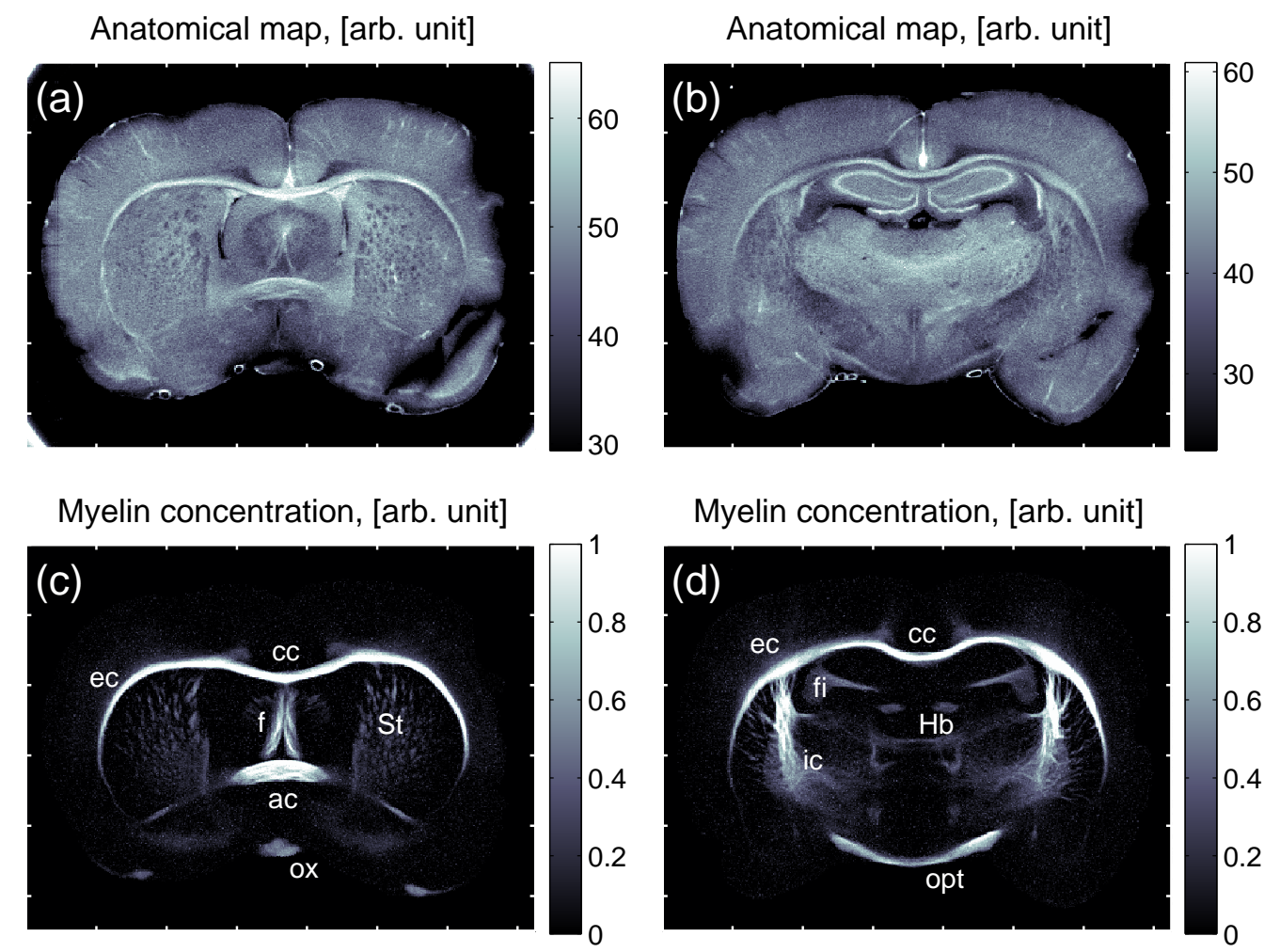

Myelin concentration, [arb. unit]

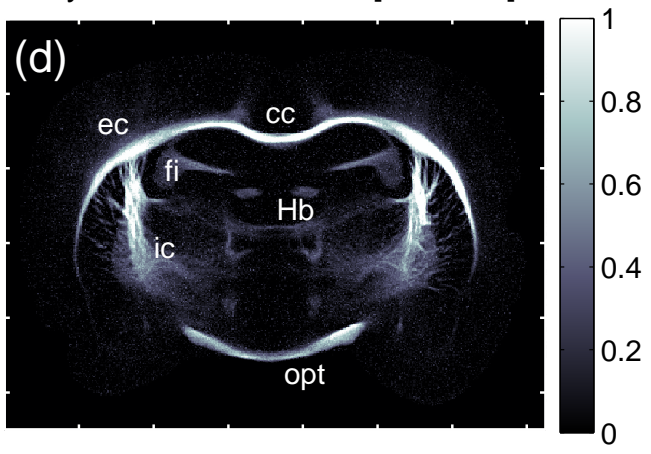

Single layer thickness, [nm]

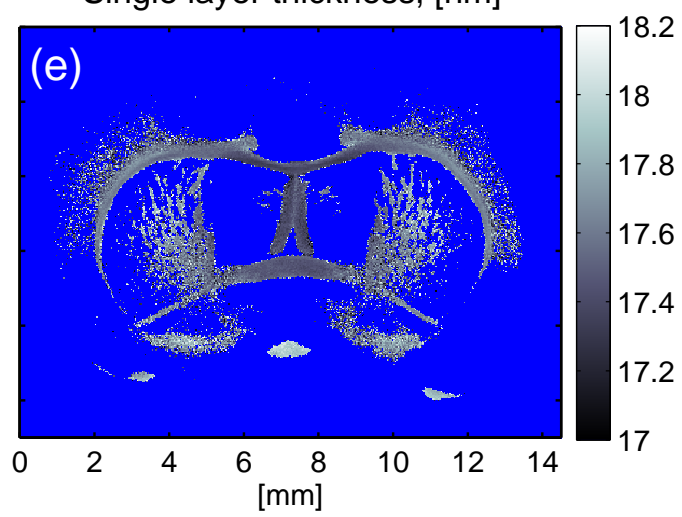

Single layer thickness, $[\mathrm{nm}]$

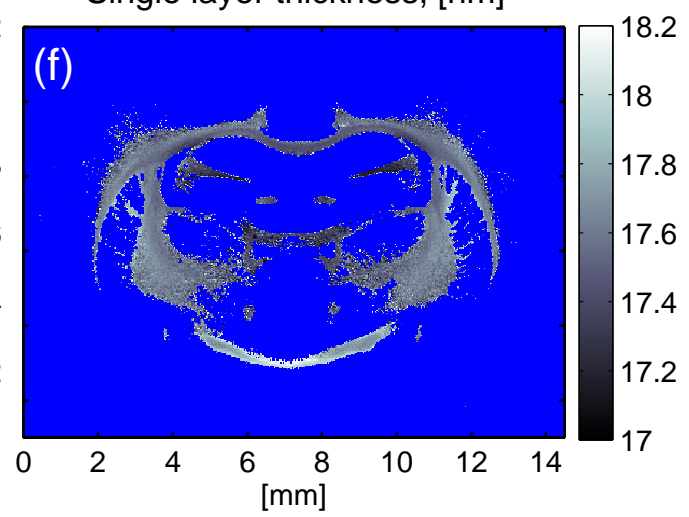

Figure 2: Myelin mapping. Two different brain slices are shown here. (a,b) The integrated scattering cross section for $q_{r}=[0.05-1.4] \mathrm{nm}^{-1}$ provides anatomical maps of the brain slices to which we can relate the myelin maps. (c,d) Maps of the concentration of myelin in each voxel determined from the intensity of the 2nd-order Bragg peaks. (e,f) Maps of the periodicity of the lamellar structure of the myelin sheaths determined from the Bragg peaks positions. The blue color refers to areas with no or very low myelin content. A selection of structural labels are shown in column 2. 
vides no information about the molecular parameters of the myelin sheaths. Demyelination and hence the concentration of myelin is important for several of the diseases mentioned above. With SAXS-CT it may be possible to quantify exactly what the concentration of myelin is in a specific volume and thus use it to study partial demyelination. Further studies are needed to investigate the quantification of the determined concentrations.

\subsection{Mapping the myelin sheath period}

The local concentration of myelin is not the only information we obtain from the fits. We also determine the positions of the Bragg peaks, which are given by the period of the myelin sheaths. Assuming the gap between the membranes does not change, the period is equivalent to the thickness of the myelin layers. Differences in period can occur both naturally and as a result of disease or treatment (Karlsson, 1966; Marta et al., 2003). In Fig. $2 \mathrm{e}, \mathrm{f}$ the variations of the period is mapped. This molecular parameters can be used to distinguish different forms of myelin in the brain. In row 1 we note that the myelin in the optic chiasms in the bottom has a period that is significantly larger than the myelin in the corpus callosum and external capsule. In row 2 we see the difference corpus callosum and the optical tract. The periodicity of the myelin sheaths can also be measured using electron microscopy (Karlsson, 1966; Marta et al., 2003). Due to the necessary high resolution of the images in electron microscopy, where each nerve fiber is imaged individually, only very limited areas can be studied at a time. The method is thus poorly suited for maps of molecular parameters over larger areas.

\subsection{Mapping the thickness of the myelin sheath}

As we determine the position of the peak we also determine the width of the peak. The width is mainly controlled by the total thickness of the myelin sheaths (finite size effect) and the periodicity variation within a single voxel (peak position variation effect) (Als-Nielsen and McMorrow, 2001). If several peaks in a series are fitted it is possible to separate the two effects. The 3rd-order peak of myelin overlaps with a peak from the neurofilament series and the 4th-order peak is in most cases not strong enough to extract useable information from. With the present data a voxel by voxel deconvolution is thus not possible. This is however not an intrinsic limitation of the method. Since the total thickness and the periodicity variation both contribute positively to the width of the peak we can determine a lower limit to the total 

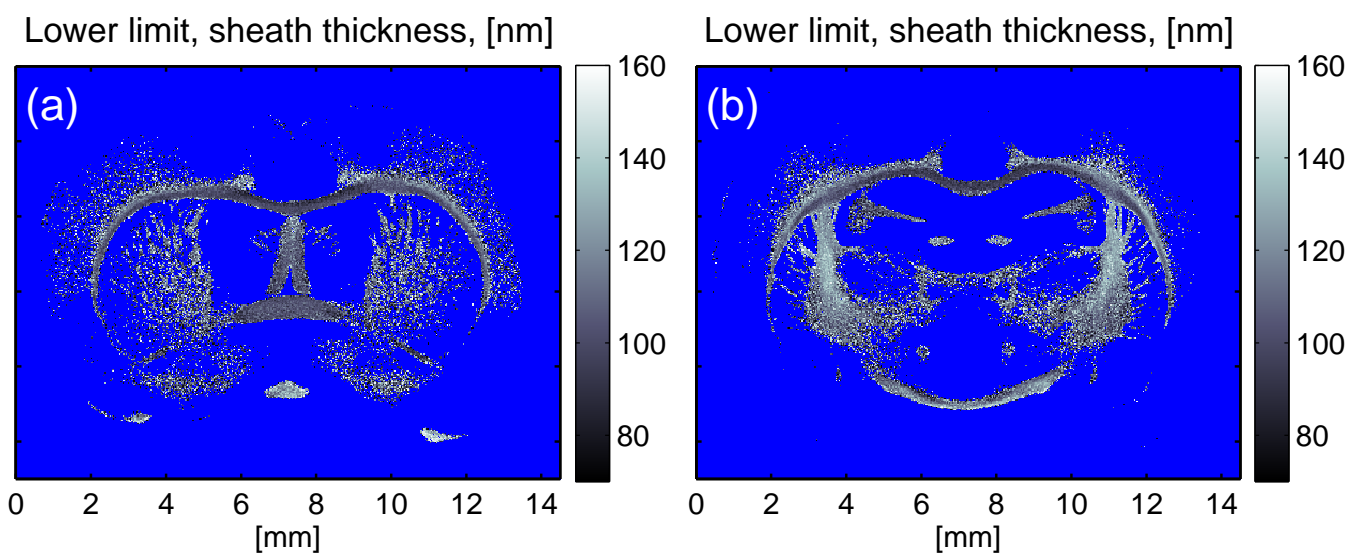

Figure 3: Thickness of myelin sheaths. (a,b) Maps of the lower boundary of the thickness of the myelin sheaths as determined from the width of the Bragg peaks. The actual thickness has in some cases been determined to be 30-50\% greater than the lower boundary. (a) and (b) corresponds to the two slices shown in Fig. 2c,d.

thickness of the myelin sheaths. These results are shown in Fig. 3a,b. From three points chosen in the corpus callosum and external capsule where the 4th-order peak is significant we determined that the actual thickness is 30$50 \%$ larger than the lower limit shown in the Fig. 3.

With the knowledge of the total thickness of the myelin sheaths it is possible to combine this with the single layer thickness to make a map of the average number myelin layers in a sheath.

The average number of layers in the myelin sheaths is very important when trying to understand how demyelination takes place (Schröder , 2005). Maps such as those shown in Fig. 3a,b would be able to tell whether demyelination take place with a part of the axons loosing all of their myelin or all of the axons loosing a part of their myelin. Deconvolution of the two signals would also provide the periodicity variation which has also been linked to treatment and diseases (Marta et al., 2003).

\subsection{Complementary molecular information}

All of the above results were deduced from the myelin Bragg peak series. With SAXS-CT a range of complementary information is automatically included. One type of information is the information available from the second series of Bragg peaks. The series probably arises from the cytoskeletal neurofilaments. The neurofilaments are a part of the axons that the myelin sheaths 

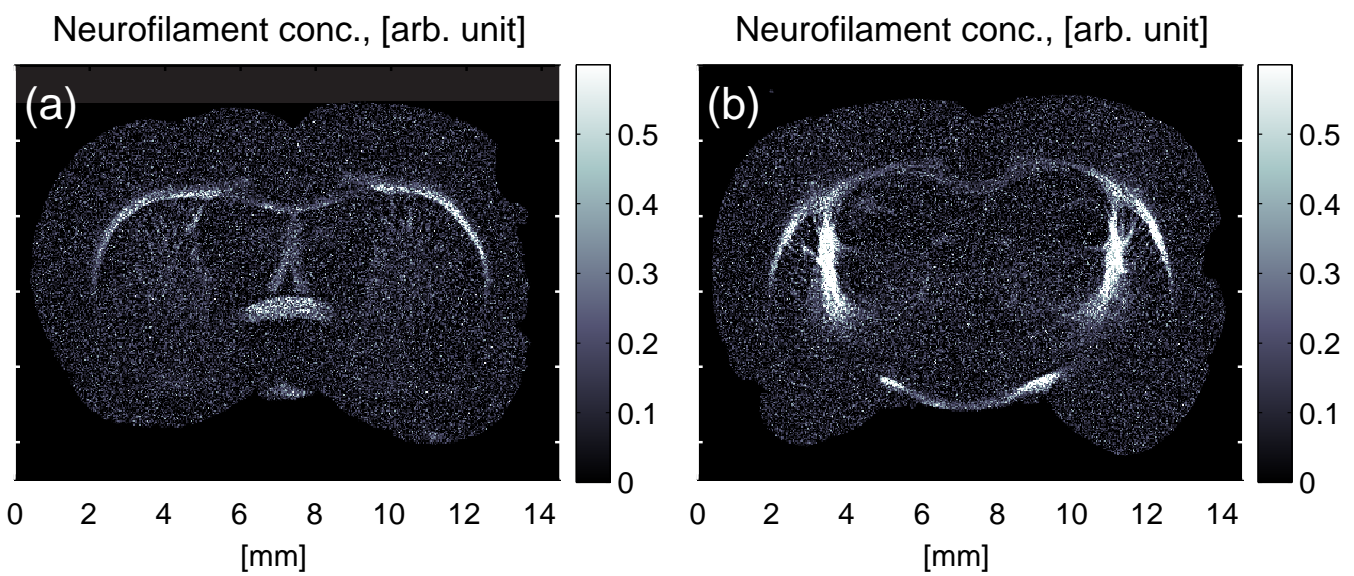

Figure 4: Density of cytoskeletal neurofilaments. (a,b) Maps of the density of cytoskeletal neurofilaments determined from the first-order Bragg peak. (a) and (b) corresponds to the two slices shown in row 1 and 2 of Fig. 2. Note that the neurofilament location is closely correlated to the location of myelin, though there are some uncorrelated variations in density, when comparing to Fig. 2c,d.

are wrapped around. Correlation between neurofilament changes and neurodegenerative diseases has demonstrated (Liu et al., 2004). Shown in Fig. $4 a, b$ are maps of the concentration of neurofilaments determined from the intensity of the 1st-order peak. We note that the location of the neurofilaments is closely correlated to the location of the myelin, as expected. However there seem to be uncorrelated variations in the concentration of the two when comparing Fig. 2b,e and Fig. 4a,b. This is also seen in Fig. 1c,d where the scattering curve from point 1 in the internal capsule shows a distinct Bragg peak from the neurofilaments while the scattering curve from point 2 in the corpus callosum shows no Bragg peak from the neurofilaments, while having similar amounts of myelin. All of the analysis made on the Bragg peak series of myelin presented above can also be made on the peaks of the neurofilaments. A full discussion of the correlations between variations of molecular parameters and different pathologies are left for future studies.

This study was performed on a rat-brain. It may be of interest to extend this method to use on human brain tissue. Due to the limitations in volume of the sample, complete human brains will be extremely difficult to study in tomographic mode. However smaller sections of a human brain could easily be imaged using this method. 


\section{Discussion}

We have in this paper demonstrated how SAXS-CT non-invasively can be used to map molecular information about the myelin sheaths in a rat brain. Using SAXS-CT we mapped the concentration and periodicity of the myelin sheaths. We also demonstrated how the method can be used to determine the local average thickness of the myelin sheaths as well as the local average periodicity variation. Finally we demonstrated that the method also provides molecular information about the cytoskeletal neurofilaments of the axons and how this information correlates with the myelin information. SAXS-CT thus provides a new and valuable tool to study myelin and improve the understanding of a number of neurodegenerative diseases.

In the future, we hope that SAXS-CT will contribute to an understanding of widespread neurodegenerative diseases such as cerebral malaria, multiple sclerosis and Alzheimer's disease where molecular parameters of myelin are important.

\section{Acknowledgements}

We gratefully acknowledge Keld Theodor and Xavier Donath for technical support, and Anders Skjolding and Casper Hempel for fruitful discussions. F.P. and M.B. acknowledges support through the DFG excellence cluster 'Munich-Centre for Advanced Photonics'. T.H.J. acknowledges support from the Danish Natural Science Research Council through DANSCATT. The experiment was performed at the cSAXS beamline of the Swiss Light Source, Paul Scherrer Institut, Villigen, Switzerland.

\section{References}

Als-Nielsen, J. and McMorrow, D. (2001). Elements of Modern X-ray Physics, John Wiley \& Sons Ltd.

Bartzokis G. Quadratic trajectories of brain myelin content: unifying construct for neuropsychiatric disorders. Neurobiol. Aging 25, 49-62 (2004).

Benda, P., Someda, K., Messer, J., \& Sweet W. H. Morphological and immunochemical studies of rat glial tumors and clonal strains propagated in culture. J. Neurosurg. 34, 310-23 (1971). 
Bunk, O. et al. Multimodal x-ray scatter imaging. New Jour. of Phys. 11, 123016 (2009).

De Felici, M. et al. Structural characterization of the human cerebral myelin sheath by small angle x-ray scattering. Phys. Med. Biol. 53, 5675-5688 (2008).

Fernandez, M. et al. Human breast cancer in vitro: Matching histo-pathology with small-angle x-ray scattering and diffraction enhanced x-ray imaging. Phys. Med. Biol. 50, 2991-3006 (2005).

Fratzl, P., Jakob, H.F., Rinnerthaler, S., Roschger, P., \& Klaushofer, K. Position-Resolved Small-Angle X-ray Scattering of Complex Biological Materials. J. Appl. Cryst. 30 765-769 (1997).

Gourrier, A. et al. Scanning X-ray imaging with small-angle scattering contrast. J. Appl. Cryst. 40, 7882 (2007).

Harding, G., Kosanetzky, J., \& Neitzel, U. Elastic Scatter computed tomography. Phys. Med. Biol. 30, 183-186 (1985).

Harding, G., Kosanetzky, J., \& Neitzel, U. X-ray diffraction computed tomography. Med. Phys. 14, 515-525 (1987).

Harding, G., \& Kosanetzky, J. Scattered x-ray beam nondestructive testing. Nucl. Inst. and methods in Physics research A A280, 517-528 (1989).

Henrich, B. et al. PILATUS: A single photon counting pixel detector for X-ray applications. Nucl Instrum Meth A 607, 247-249 (2009).

Hui, E.S., Cheung, M.M., Chan, K.,\& Wu, E.X. B-value dependence of DTI quantitation and sensitivity in detecting neural tissue changes. Neuroimage 49, 2366-2374 (2010).

Janota, I. \& Doshi, I. Cerebral malaria in the United Kingdom. J. Clin. Pathol. 32, 769-772 (1979).

Kak, A. C., \& Slaney, M. Principles of Computerized Tomographic Imaging (IEEE Press, New York, 1988).

Karlsson, U. Comparison of the Myelin Period of Peripheral and Central Origin by Electron Microscopy. J. Ultra. Res. 15, 451-468 (1966). 
Kirschner, D. A. et al. Rapid Assessment of Internodal Myelin Integrity in Central Nervous System Tissue. Journal of Neuroscience Research 88, 712723 (2010).

Kleuker, U., Suortti, P., Weyrich, W., \& Spanne, P. Feasibility study of x-ray diffraction computed tomography for medical imaging. Phys. Med. Biol. 43, 2911-2923 (1998).

Lareida, A., Beckmann, F., Schrott-Fischer, A., Glueckert, R., Freysinger, W., \& Müller B. High-resolution X-ray tomography of the human inner ear: synchrotron radiation-based study of nerve fibre bundles, membranes and ganglion cells. J. Microsc., 234, 1, 95-102 (2009).

Liu, Q. et al. Neurofilament proteins in neurodegenerative diseases. Cell. Mol. Life Sci. 61, 3057-3075 (2004).

Marta, C. B. et al. Morphological changes of myelin sheaths in rats intracranially injected with apotransferrin. Neurochem. Res. 28, 101-110 (2003).

Mikula, S., Trotts, I., Stone, J. M. \& Jones, E. G. Internet-enabled highresolution brain mapping and virtual microscopy. NeuroImage 35, 1, 9-15 (2007).

Müller, B., Deyhle, H., Bradley, D., Farquharson, M., Schulz, G., MüllerGerbl, M., \& Bunk, O.Scanning x-ray scattering: Evaluating the nanostructure of human tissues. European Journal of Clinical Nanomedicine 3, 30-33 (2010).

Paris, O., Zizak, I., Lichtenegger, H., Roschger, P., Klaushofer, K., \& Fratzl, P. Analysis of the hierarchical structure of biological tissues by scanning x-ray scattering using a micro-beam. Cell. Mol. Biol. 46, 993-1004 (2000).

Paxinos, G. \& Watson, C. eds. The rat brain in stereotaxic coordinates (New York: Academic Press, 1986).

Peterson, J.W., Bö, L., Mörk, S., Chang, A.,\& Trapp, B.D. Transected Neurites, Apoptotic Neurons, and Reduced Inflammation in Cortical Multiple Sclerosis Lesions. Ann. Neurol. 50, 389-400 (2001).

Prineas, J.W. The neuropathology of multiple sclerosis. In: Koetsier J.C., ed. Handbook of clinical neurology. Demyelinating disease, vol 3. (Amsterdam, Elsevier, 1985) 213257. 
Regnard, P. et al. Irradiation of intracerebral 9L gliosarcoma by a single array of microplanar x-ray beams from a synchrotron: balance between curing and sparing. Phys. Med. Biol. bf 53, 4, 861-78 (2008).

Rinnerthaler, S., Roschger, P., Jakob, H.F., Nader, A., Klaushofer, K., \& Fratzl, P. Scanning small angle x-ray scattering analysis of human bone sections. Calcif. Tissue Int. 64, 422-429 (1999).

Schröder, J. M. Neuropathology of Charcot-Marie-Tooth and Related Disorders. NeuroMolecular Medicine 8, 23-42 (2005).

Schroer, C. G. et al. Mapping the local nanostructure inside a specimen by tomographic small-angle x-ray scattering. Appl. Phys. Lett. 88, 164102 (2006).

Sjöstrand, F. S., The lamellated structure of the nerve myelin sheath as revealed by high-resolution electron microscopy. Experientia 9, 68-69 (1953).

Stock, S. R., de Carlo, F., \& Almer, J. D. High energy X-ray scattering tomography applied to bone. J. Struct. Biol. 161, 144-150 (2008).

Tartari, A., De Felici, M., Gambaccini, M., Querzoli, P., Bonifazzi, C., Ferrerod, C., \& Felici, R. Biological tissue characterisation in narrow beam x-ray scattering techniques. X-Ray Spectrom. 38, 195199 (2009).

Trapp, B.D. et al. Axonal transection in the lesions of multiple sclerosis. $N$. Eng. J. Med. 338, 278-285 (1998). 